\title{
VOGUE: Answer Verbalization through Multi-Task Learning
}

\author{
Endri Kacupaj ${ }^{1}$, Shyamnath Premnadh ${ }^{1}$, Kuldeep Singh ${ }^{2,3}$, Jens Lehmann ${ }^{1,4}$ \\ Maria Maleshkova ${ }^{1}$ \\ ${ }^{1}$ University of Bonn, ${ }^{2}$ Zerotha Research, ${ }^{3}$ Cerence GmbH, ${ }^{4}$ Fraunhofer IAIS \\ \{kacupaj, jens.lehmann, maleshkova\}ecs.uni-bonn. de \\ s6shpremeuni-bonn.de \\ kuldeep.singhl@cerence.com \\ jens.lehmann@iais.fraunhofer.de
}

\begin{abstract}
In recent years, there have been significant developments in Question Answering over Knowledge Graphs (KGQA). Despite all the notable advancements, current KGQA systems only focus on answer generation techniques and not on answer verbalization. However, in real-world scenarios (e.g., voice assistants such as Alexa, Siri, etc.), users prefer verbalized answers instead of a generated response. This paper addresses the task of answer verbalization for (complex) question answering over knowledge graphs. In this context, we propose a multi-task-based answer verbalization framework: VOGUE (Verbalization thrOuGh mUlti-task lEarning). The VOGUE framework attempts to generate a verbalized answer using a hybrid approach through a multitask learning paradigm. Our framework can generate results based on using questions and queries as inputs concurrently. VOGUE comprises four modules that are trained simultaneously through multi-task learning. We evaluate our framework on all existing datasets for answer verbalization, and it outperforms all current baselines on both BLEU and METEOR scores as evaluation metric.
\end{abstract}

\section{Introduction}

In recent years, publicly available knowledge graphs (KG) (e.g., DBpedia (Lehmann et al., 2015), Wikidata (Vrandečić and Krötzsch, 2014)) have been broadly adopted as a source of knowledge in several tasks such as entity linking, relation extraction, and question answering (Kacupaj et al., 2021b). Question answering (QA) over knowledge graphs, in particular, is an essential task that maps a user's utterance to a query over a KG to retrieve the correct answer (Singh et al., 2018). The initial knowledge graph question answering systems (KGQA) were mostly templateor rule-based systems with limited learnable mod-

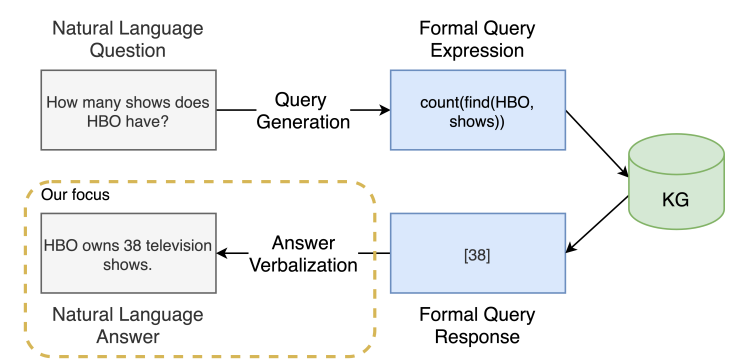

Figure 1: A QA pipeline with integrated answer verbalization module. Our focus is the answer verbalization task as we assume logical form is generated by a QA system using the input question.

ules (Unger et al., 2012). With the increasing popularity of intelligent personal assistants (e.g., Alexa, Siri), the research focus has been shifted to conversational question answering over KGs (ConvQA) that involve single-turn/multi-turn dialogues (Kacupaj et al., 2021a).

Existing open-source KGQA systems are restricted to only generating or producing answers without verbalizing them in natural language ( $\mathrm{Fu}$ et al., 2020). The lack of verbalization makes the interaction with the user not natural in contrast to voice assistants such as Siri and Alexa. Figure 1 depicts an ideal integration of a QA pipeline with answer verbalization. For instance, assuming that the answer to the exemplary question, "How many shows does HBO have?" is not known by the user. Suppose the QA system only responds with a number (e.g., 38) as an answer (similar to open-source KGQA systems), with no further explanation. In that case, the user might need to refer to an external data source to verify the answer. In an attempt to enable the users to verify the answer provided by a QA system, researchers employed techniques such as (i) revealing the generated formal query (Ferré, 2017), (ii) graphical visualizations of the formal query (Zheng et al., 2017) and (iii) verbalizing the formal query (Ell et al., 2014). Understanding the necessity of verbalized answers 
in the KGQA domain, recently, several datasets have been proposed (Kacupaj et al., 2020; Biswas et al., 2021). For the answer verbalization task, the system has to verbalize the answer to convey not only the information requested by the user but also additional characteristics that indicate how the answer was determined. In our exemplary question (from Kacupaj et al. (2021a) dataset), a verbalized response would look like, "HBO owns 38 television shows." or "There are $38 \mathrm{TV}$ shows whose owner is HBO.". Both answers allow the user to verify that the system retrieved the total number of TV shows owned by HBO. In the literature, there exist empirical results showing that answer verbalization quantitatively and qualitatively improves the ability to understand the answer (Kacupaj et al., 2021a). However, it remains an open question - How can we verbalize an answer, given a logical form and an input question. With our work we address precisely this open and highly relevant research question.

In this paper, we propose VOGUE, the first approach dedicated to verbalize answers for KGQA. Our idea is to employ the question (user utterance) and the QA system-generated query as inputs. We refer to this strategy as "hybrid", since the final verbalized answer is produced using both the question and query concurrently. This work argues that leveraging content from both sources allows the model for better convergence and provides new, improved results. Furthermore, we complement our hypothesis with multi-task learning paradigms, since multi-task learning has been quite efficient for different system architectures (Cipolla et al., 2018), including question answering systems (Plepi et al., 2021; Kacupaj et al., 2021b). Our framework can receive two (e.g., question \& query) or even one (e.g., question) input. It consists of four modules that are trained simultaneously to generate the verbalized answer. The first module employs a dual transformerbased encoder architecture for encoding the inputs. The second module determines whether the encoded inputs are relevant and decides if both will be used for verbalization. The third module consists of a cross-attention network that performs question and query matching by jointly modeling the relationships of question words and query actions. The final module employs a transformer decoder that is used to generate the final verbalization. Our work makes the following contributions:
- We introduce the first multi-task-based hybrid answer verbalization framework consisting of simultaneously trained four modules.

- We propose a similarity threshold and cross attention modules to determine the relevance between the inputs and fuse information to employ a hybrid strategy.

- We provide an extensive evaluation and ablation study of the proposed framework on three QA datasets with answer verbalization. Our evaluation results establish a new baseline for answer verbalization task, which we believe will drive future research in a newly studied problem.

To facilitate reproducibility and reuse, our framework implementation is publicly available ${ }^{1}$. The structure of the paper is as follows: Section 2 summarizes the related work. Section 3 provides the task definition. Section 4 presents the proposed framework. Section 5 describes the experiments, results, ablation study and error analysis. We conclude in Section 6.

\section{Related Work}

As part of the related work we describe previous efforts and refer to different approaches from research fields, including task-oriented dialog systems, WebNLG, and KGQA systems.

A task-oriented dialogue system aims to help the user complete certain tasks in a specific domain (e.g. restaurant booking, weather query, or flight booking), making it valuable for realworld business. Typically, task-oriented dialogue systems are built on top of a structured ontology, which defines the tasks' domain knowledge. Work in (Bordes et al., 2017) formalized the taskoriented dialogue as a reading comprehension task regarding the dialogue history as context, user utterance as the question, and system response as the answer. In their work, authors utilized end-to-end memory networks for multi-turn inference. In (Lei et al., 2018), authors proposed a two-step seq 2 seq generation model, which bypassed the structured dialogue act representation and only retain the dialogue state representation. Kassawat et al. (2019) proposed RNN-based end-to-end encoder-decoder architecture, which employs joint embeddings of the knowledge graph and the corpus as input.

\footnotetext{
${ }^{1}$ https://github.com/endrikacupaj/VOGUE
} 
The WebNLG is a challenge that consists of mapping structured data to a textual representation. The dataset (Gardent et al., 2017) contains data/text pairs where the data is a set of triples extracted from DBpedia, and the text is the verbalization of these triples. The dataset has been promoted for the development of 1) RDF verbalizers and 2) microplanners to handle a wide range of linguistic constructions. In our case, we only focus on related work pertaining to RDF verbalizers. Zhao et al. (2020) propose DualEnc, a dual encoding model that can incorporate the graph structure and cater to the linear structure of the output text. Song et al. (2020) proposes a graph-totext approach that leverages richer training signals to guide the model for preserving input information. They introduce two types of autoencoding losses, each individually focusing on different aspects of input graphs. The losses are then backpropagated to calibrate the model via multi-task training. Work in Liu et al. (2020) propose an attention-based model, which mainly contains an entity extraction module and a relation detection module. The model devises a supervised multihead self-attention mechanism as the relation detection module to learn the token-level correlation for each relation type separately.

The task-oriented dialogue systems and WebNLG contain various approaches for generating text; nevertheless, none of them can be applied directly to solve answer verbalization for KGQA systems. Most task-oriented dialogue systems are designed and implemented to fit their corresponding task, and therefore they would not be suitable for open-domain knowledge graphs (e.g. Wikidata, DBpedia). Regarding WebNLG, the task only considers triples or graph structure data as input. In answer verbalization, the model input can be the question and/or the query. While the query can be translated into a graph structure, there is no support for textual information such as the question.

\section{Task Definition}

In this work, we target the problem of answer verbalization for KGQA. A semantic parsing-based QA system maps the question into executable logical forms and then executes it on a KG to produce the answer. For our task, given the question, the generated logical form, and the extracted answer, we aim to generate a natural language sen- tence, with the requirements that it is grammatically sound and correctly represents all the information in the question, logical form, and answer. Formally, let $X, Y$ denote the source-target pair. $X$ contains the set of questions, logical forms, answers, and $Y$ corresponds to $y_{1}, y_{2}, \ldots, y_{m}$, which is the verbalized answer of $X$. The goal of the answer verbalization is to learn a distribution $p(Y \mid X)$ to generate natural language text describing the answer automatically.

\section{Approach}

In question answering, the input data consists of question $u$ and its answer $a$, extracted from the knowledge graph. The QA system will map the question to a logical form $z$ depending on the context. For answer verbalization, VOGUE maps the question, logical form, and answer to natural language sentence $s$. Figure 2 shows the architecture of VOGUE.

\subsection{Dual Encoder}

To encode both the question and logical form, we employ a dual encoder architecture. Our dual encoder consists of two instances of the transformer encoder (Vaswani et al., 2017).

First, as a preprocessing, we use a previous competitive pre-trained named entity recognition model (Yamada et al., 2020) to identify and replace all entities in the question with a more general entity token $[E N T]$. In this way, we allow our model to focus on the sentence structure and relations between words. Furthermore, our model learns the positions of entities in the question. It also allows VOGUE to predict the respective entity positions in the verbalized answer. The same preprocessing step applies to the logical form. At the end of each input, we append a context token $[C T X]$, which is used later as a semantic representation.

Next, given the question utterance $q$ containing $n$ words $\left\{w_{1}, \ldots, w_{n}\right\}$ and the logical form $l$ containing $m$ actions $\left\{a_{1}, \ldots, a_{m}\right\}$, we tokenize the contexts and use the pre-trained model GloVe (Pennington et al., 2014) to embed the words into a vector representation space of dimension $d^{2}$. Our word embedding model provides us with the sequences $x^{(q)}=\left\{x_{1}^{(q)}, \ldots, x_{n}^{(q)}\right\}$, $x^{(l f)}=\left\{x_{1}^{(l f)}, \ldots, x_{m}^{(l f)}\right\}$ where $x_{i}^{(q)}, x_{i}^{(l f)}$ are

\footnotetext{
${ }^{2}$ We employ the same dimension $d$ for all the representations, unless it is explicitly mentioned.
} 


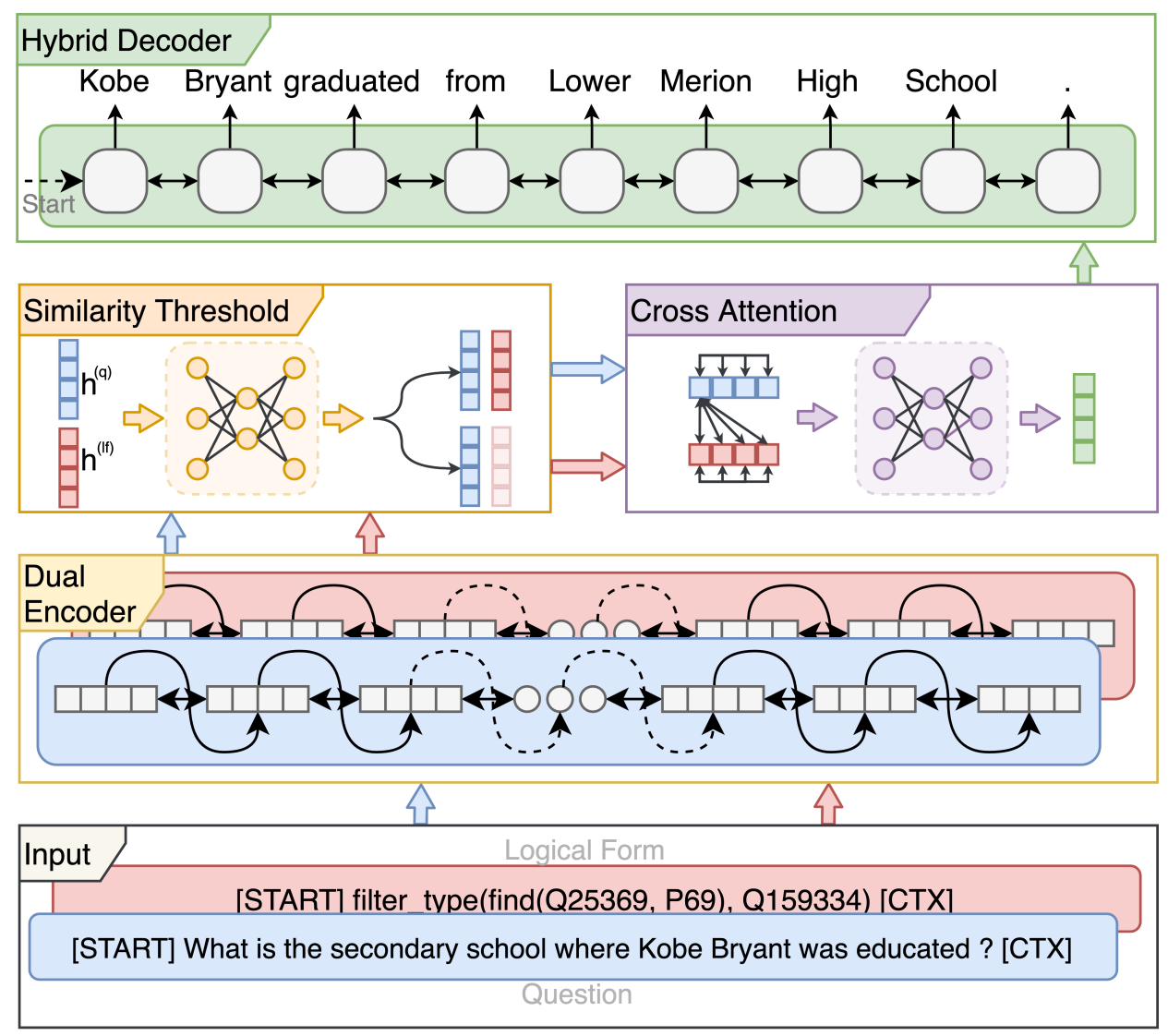

Figure 2: VOGUE's architecture. It consists of four modules: 1) A dual encoder that is responsible to encode both inputs (question, logical form). 2) A similarity threshold module that determines whether the encoded inputs are relevant and determines if both will be used for verbalization. 3) A cross-attention module that performs question and query matching by jointly modeling the relationships of question words and query actions. 4) A hybrid decoder that generates the verbalized answer using the information of both question and logical form representations from the cross-attention module.

given by,

$$
\begin{aligned}
& x_{i}^{(q)}=\operatorname{GloVe}\left(w_{i}\right), \\
& x_{i}^{(l f)}=\operatorname{GloVe}\left(a_{i}\right),
\end{aligned}
$$

and $x_{i}^{(q)}, x_{i}^{(l f)} \in \mathbb{R}^{d}$. Afterwards, both sequences are forwarded through the transformer encoders. The two encoders here output the contextual embeddings $h^{(q)}=\left\{h_{1}^{(q)}, \ldots, h_{n}^{(q)}\right\}$ and $h^{(l f)}=$ $\left\{h_{1}^{(l f)}, \ldots, h_{m}^{(l f)}\right\}$, where $h_{i}^{(q)}, h_{i}^{(l f)} \in \mathbb{R}^{d}$. We define this as:

$$
\begin{aligned}
& h^{(q)}=\operatorname{encoder}_{q}\left(x_{q} ; \theta^{\left(e n c_{q}\right)}\right), \\
& h^{(l f)}=\operatorname{encoder}_{l f}\left(x_{l f} ; \theta^{\left(\operatorname{enc}_{l f}\right)}\right),
\end{aligned}
$$

where $\theta^{\left(e n c_{q}\right)}, \theta^{\left(e n c_{l f}\right)}$ are the encoders trainable parameters.

\subsection{Similarity Threshold}

Given the encoded question utterance and logical form, VOGUE's second module is responsible for learning the relevance between the inputs and de- termining whether we will employ both for verbalization. This module is necessary when we want to utilize our framework alongside a question answering system. If we assume that the QA system is perfect and always produces correct logical forms, this module can be skipped. However, in a real-world scenario, QA systems are far from perfect. Therefore, we employ this module, which intends to identify the threshold for determining if two inputs are similar or not. The input here is the concatenation of the hidden states of the encoded question utterance $h^{(q)}$ and logical form $h^{(l f)}$. The module will perform binary classification on the vocabulary $V^{(s t)}=\{0,1\}$, where 0 indicates that there is no high relevance between the inputs, and only the question will be used for verbalization. While 1 allows us to use both and continue with the next module. Overall, our similarity threshold module is implemented using two linear layers, a Leaky ReLU activation function and a softmax for 
the predictions. Formally we define the module as:

$$
\begin{aligned}
& h^{(s t)}=\operatorname{LeakyReLU}\left(\boldsymbol{W}^{\left(s t_{1}\right)}\left[h^{(q)} ; h^{(l f)}\right]\right), \\
& p^{(s t)}=\operatorname{softmax}\left(\boldsymbol{W}^{\left(s t_{2}\right)} h^{(s t)}\right),
\end{aligned}
$$

where $\boldsymbol{W}^{\left(s t_{1}\right)} \in \mathbb{R}^{d \times 2 d}$ are the weights of the first linear layer and $h^{(s t)}$ is the hidden state of the module. $\boldsymbol{W}^{\left(s t_{2}\right)} \in \mathbb{R}^{\left|V^{(s t)}\right| \times d}$ are the weights of the second linear layer, $\left|V^{(s t)}\right|$ is the size of the vocabulary and $p^{(s t)}$ denotes the probability distribution over the vocabulary indices.

\subsection{Cross Attention}

Inspired by recent computer vision research (Wei et al., 2020; Mohla et al., 2020), we employ a cross-attention module that exploits relationships between the inputs and fuses information. The module here performs question and logical form matching by jointly modeling the relationships of question words and logical form actions. Our cross-attention approach is a variation of the self-attention mechanism (Vaswani et al., 2017). In the self-attention mechanism the output is determined by a query and a set of key-value pairs. Given the stacked encoded question and logical form, $h^{(q l f)}=\left(\begin{array}{l}h^{(q)} \\ h^{(l f)}\end{array}\right)=$ $\left\{h_{1}^{(q)}, \ldots, h_{n}^{(q)} ; h_{1}^{(l f)}, \ldots, h_{m}^{(l f)}\right\}$, where $h^{(q l f)} \in$ $\mathbb{R}^{2 \times d}$ we calculate the query and key-value pairs using three linear projections:

$$
\begin{aligned}
& \boldsymbol{Q}^{(q l f)}=\boldsymbol{W}^{(Q)} h^{(q l f)}=\left(\begin{array}{c}
\boldsymbol{W}^{(Q)} h^{(q)} \\
\boldsymbol{W}^{(Q)} h^{(l f)}
\end{array}\right)=\left(\begin{array}{c}
\boldsymbol{Q}^{(q)} \\
\boldsymbol{Q}^{(l f)}
\end{array}\right), \\
& \boldsymbol{K}^{(q l f)}=\boldsymbol{W}^{(K)} h^{(q l f)}=\left(\begin{array}{c}
\boldsymbol{W}^{(K)} h^{(q)} \\
\boldsymbol{W}^{(K)} h^{(l f)}
\end{array}\right)=\left(\begin{array}{c}
\boldsymbol{K}^{(q)} \\
\boldsymbol{K}^{(l f)}
\end{array}\right), \\
& \boldsymbol{V}^{(q l f)}=\boldsymbol{W}^{(V)} h^{(q l f)}=\left(\begin{array}{c}
\boldsymbol{W}^{(V)} h^{(q)} \\
\boldsymbol{W}^{(V)} h^{(l f)}
\end{array}\right)=\left(\begin{array}{c}
\boldsymbol{V}^{(q)} \\
\boldsymbol{V}^{(l f)}
\end{array}\right),
\end{aligned}
$$

where $\quad \boldsymbol{W}^{(Q)}, \boldsymbol{W}^{(K)}, \boldsymbol{W}^{(V)} \in \mathbb{R}^{d \times d}$ are the weights of the linear layers and $\boldsymbol{Q}^{(q l f)}, \boldsymbol{K}^{(q l f)}, \boldsymbol{V}^{(q l f)}$ are the query, key and value of the stacked question and logical form. Next, for calculating the cross-attention we simplify the "Scaled Dot-Product Attention" (Vaswani et al., 2017) step by removing the scaling factor and softmax. We end-up calculating the attention of our input as described below:

$$
\begin{aligned}
& \operatorname{Attention}\left(\boldsymbol{Q}^{(q l f)}, \boldsymbol{K}^{(q l f)}, \boldsymbol{V}^{(q l f)}\right) \\
& =\boldsymbol{Q}^{(q l f)} \boldsymbol{K}^{(q l f) T} \cdot \boldsymbol{V}^{(q l f)} \\
& =\left(\begin{array}{c}
\boldsymbol{Q}^{(q)} \\
\boldsymbol{Q}^{(l f)}
\end{array}\right)\left(\boldsymbol{K}^{(q) T} \boldsymbol{K}^{(l f) T}\right) \cdot\left(\begin{array}{c}
\boldsymbol{V}^{(q)} \\
\boldsymbol{V}^{(l f)}
\end{array}\right) \\
& =\left(\begin{array}{cc}
\boldsymbol{Q}^{(q)} \boldsymbol{K}^{(q) T} & \boldsymbol{Q}^{(q)} \boldsymbol{K}^{(l f) T} \\
\boldsymbol{Q}^{(l f)} \boldsymbol{K}^{(q) T} & \boldsymbol{Q}^{(l f)} \boldsymbol{K}^{(l f) T}
\end{array}\right) \cdot\left(\begin{array}{c}
\boldsymbol{V}^{(q)} \\
\boldsymbol{V}^{(l f)}
\end{array}\right) \\
& =\left(\begin{array}{c}
\boldsymbol{Q}^{(q)} \boldsymbol{K}^{(q) T} \boldsymbol{V}^{(q)}+\boldsymbol{Q}^{(q)} \boldsymbol{K}^{(l f) T} \boldsymbol{V}^{(l f)} \\
\boldsymbol{Q}^{(l f)} \boldsymbol{K}^{(l f) T} \boldsymbol{V}^{(l f)}+\boldsymbol{Q}^{(l f)} \boldsymbol{K}^{(q) T} \boldsymbol{V}^{(q)}
\end{array}\right) .
\end{aligned}
$$

While calculating the cross-attention for the question, we also use the key-value pair from the logical form $\left(\boldsymbol{K}^{(l f)}, \boldsymbol{V}^{(l f)}\right)$, the same applies when calculating the cross-attention for the logical form. After calculating the cross-attentions, we use the same steps as in the transformer to produce the new representations for our inputs. Finally, considering $h^{(q c a)}, h^{(l f c a)}$ the output representations of the cross-attention module for the question and logical form respectively, we concatenate them and forward them to the hybrid decoder module.

\subsection{Hybrid Decoder}

To translate the input question and logical form into a sequence of words (verbalized answer), we utilize a transformer decoder architecture (Vaswani et al., 2017), which employs the multi-head attention mechanism. The decoder will generate the final natural language answer. The output here is dependent on the cross-attention embedding $h^{(c a)}$. Here we define the decoder vocabulary as

$$
\begin{array}{r}
V^{(\text {dec })}=V^{(v t)} \cup\{[ \\
{[S T A R T],[E N D],} \\
[E N T],[A N S]\},
\end{array}
$$

where $V^{(v t)}$ is the vocabulary with all the distinct tokens from our verbalizations. As we can see, the decoder vocabulary contains four additional helper tokes, where two of them ([START], $[E N D])$ indicate when the decoding process starts and ends, while the other two ([ENT], $[A N S]$ ) are used to specify the position of the entities and the answer on the final verbalized sequence. On top of the decoder stack, we employ a linear layer alongside a softmax to calculate each token's probability scores in the vocabulary. We define the 
decoder stack output as follows:

$$
\begin{aligned}
& h^{(d e c)}=\operatorname{decoder}\left(h^{(c a)} ; \theta^{(d e c)}\right), \\
& p_{t}^{(d e c)}=\operatorname{softmax}\left(\boldsymbol{W}^{(d e c)} h_{t}^{(d e c)}\right),
\end{aligned}
$$

where $h_{t}^{(d e c)}$ is the hidden state in time step $t, \theta^{(d e c)}$ are the decoder trainable parameters, $\boldsymbol{W}^{(d e c)} \in \mathbb{R}^{\left|V^{(d e c)}\right| \times 2 d}$ are the linear layer weights, and $p_{t}^{(d e c)} \in \mathbb{R}^{\left|V^{(d e c)}\right|}$ is the probability distribution over the decoder vocabulary in time step $t$. The $\left|V^{(d e c)}\right|$ denotes the vocabulary size of the decoder module in VOGUE.

\subsection{Learning}

The framework consists of four trainable modules. However, we apply a loss function only on two of them (similarity threshold and hybrid decoder). The dual encoder and cross-attention modules are trained based on the similarity threshold and hybrid decoder's signal. To account for multitasking, we perform a weighted average of all the single losses:

$$
L=\lambda_{1} L^{s t}+\lambda_{2} L^{d e c},
$$

where $\lambda_{1}, \lambda_{2}$ are the relative weights learned during training considering the difference in magnitude between losses by consolidating the log standard deviation (Armitage et al., 2020; Cipolla et al., 2018). $L^{s t}$ and $L^{d e c}$ are the respective negative log-likelihood losses of the similarity threshold and hybrid decoder modules. These losses are defined as:

$$
\begin{aligned}
& L^{s t}=-\sum_{j=1}^{2 d} \log p\left(y_{j}^{(s t)} \mid x\right), \\
& L^{d e c}=-\sum_{k=1}^{m} \log p\left(y_{k}^{(d e c)} \mid x\right),
\end{aligned}
$$

where $m$ is the length of the gold logical form. $y_{j}^{(s t)} \in V^{(s t)}$ are the gold labels for the similarity threshold and $y_{k}^{(d e c)} \in V^{(d e c)}$ are the gold labels for the decoder. The model benefits from each module's supervision signals, which improves the performance in the given task.

\section{Experiments}

\subsection{Experimental Setup}

Datasets We perform experiments on three answer verbalization datasets (cf., Table 1). Below we provide a brief description of these:

\begin{tabular}{l|ccccc}
\hline Dataset & Train & Test & Ques len. & Ans len. & Vocab. \\
\hline VQuAnDa & 4000 & 1000 & 12.27 & 16.95 & 10431 \\
ParaQA & 12637 & 3177 & 12.27 & 17.06 & 12755 \\
VANiLLa & 85729 & 21433 & 8.96 & 8.98 & 50505 \\
\hline
\end{tabular}

Table 1: Dataset statistics, including the (average) number of tokens per question sentence, the (average) number of tokens per answer sentence and the vocabulary list size.

- VQuAnDa (Kacupaj et al., 2020) is the first QA dataset, which provides the verbalization of the answer in natural language. It contains 5000 "complex" questions with their SPARQL queries and answers verbalization. The dataset consists of 5042 entities and 615 relations.

- ParaQA (Kacupaj et al., 2021a) is a QA dataset with multiple paraphrased responses. The dataset was created using a semiautomated framework for generating diverse paraphrasing of the answers using techniques such as back-translation. It contains 5000 "complex" question-answer pairs with a minimum of two and a maximum of eight unique paraphrased responses for each question.

- VANiLLa (Biswas et al., 2021) is a QA dataset that offers answers in natural language sentences. The answer sentences in this dataset are syntactically and semantically closer to the question than the triple fact. The dataset consists of over 100k "simple" questions.

Model Configuration For simplicity, to represent the logical forms, we employ the same grammar as in (Kacupaj et al., 2021b). Our approach can be used with any other grammar or even directly with SPARQL queries. However, we believe it is better to employ semantic grammar from a stateof-the-art QA model. To properly train the similarity threshold module, we had to introduce negative logical forms for each question. We did that by corrupting the gold logical forms, either by replacing a random action or finding another "similar" logical form from the dataset based on the Levenshtein distance. For all the modules in our framework, we employ an embedding dimension of 300. A transformer encoder and decoder having two layers and six heads for the multi-head attention model is used. We apply dropout (Srivastava et al., 2014) with a probability 0.1. For the optimization, we use the Noam optimizer proposed 


\begin{tabular}{l|c|c|c|cc|c}
\hline \multirow{2}{*}{\multicolumn{1}{c}{ Models }} & \multicolumn{3}{c}{ BLEU } & \multicolumn{3}{c}{ METEOR } \\
\cline { 2 - 7 } & VQuAnDa & ParaQA & VANiLLa & VQuAnDa & ParaQA & VANiLLa \\
\hline RNN (Luong et al., 2015) (Q) & 15.43 & 22.45 & 16.66 & 53.15 & 58.41 & 58.67 \\
RNN (Luong et al., 2015) (LF) & 20.19 & 26.36 & 16.45 & 57.06 & 61.87 & 55.34 \\
Convolutional (Gehring et al., 2017) (Q) & 21.32 & 25.94 & 15.42 & 57.54 & 60.82 & 61.14 \\
Convolutional (Gehring et al., 2017) (LF) & 26.02 & 31.89 & 16.89 & 64.30 & 65.85 & 58.72 \\
Transformer (Vaswani et al., 2017) (Q) & 18.37 & 23.61 & 30.80 & 56.83 & 59.63 & 62.16 \\
Transformer (Vaswani et al., 2017) (LF) & 23.18 & 28.01 & 28.12 & 60.17 & 63.75 & 59.01 \\
BERT (Devlin et al., 2019) (Q) & 22.78 & 26.12 & 31.32 & 59.28 & 62.59 & 62.96 \\
BERT (Devlin et al., 2019) (LF) & 26.48 & 30.31 & 30.11 & 65.92 & 65.92 & 59.27 \\
\hline VOGUE (Ours) (H) & $\mathbf{2 8 . 7 6}$ & $\mathbf{3 2 . 0 5}$ & $\mathbf{3 5 . 4 6}$ & $\mathbf{6 7 . 2 1}$ & $\mathbf{6 8 . 8 5}$ & $\mathbf{6 5 . 0 4}$ \\
\hline
\end{tabular}

Table 2: Results on answer verbalization. VOGUE outperforms all existing baselines and achieves the new state of the art for both the BLEU and METEOR scores. The baseline experiment results are reported with two inputs: Question (Q) and gold Logical Form (LF), while VOGUE employs a Hybrid (H) approach.

by (Vaswani et al., 2017), where authors use an Adam optimizer (Kingma and Ba, 2015) with several warmup steps for the learning rate.

Model for Comparison We compare our framework with the four baselines that have been evaluated on the considered datasets. All baselines consist of sequence to sequence architectures, a family of machine learning approaches used for language processing and often used for natural language generation tasks. The first model consists of an RNN (Luong et al., 2015) based architecture, the second uses a convolutional network (Gehring et al., 2017), the third employs a transformer network (Vaswani et al., 2017), while the last one uses pre-trained BERT (Devlin et al., 2019) model. For a fair comparison with our framework, we report the baselines' results using the question and the logical form as separate inputs considering that baselines are limited to accept both inputs together.

Evaluation Metrics We use the same metrics as employed by the authors of the three existing datasets (Kacupaj et al., 2020, 2021a; Biswas et al., 2021) on the previously mentioned baselines. The BLEU score, as defined by (Papineni et al., 2002), analyzes the co-occurrences of ngrams in the reference and the proposed responses. It computes the n-gram precision for the whole dataset, which is then multiplied by a brevity penalty to penalize short translations. We report results for BLEU-4. The METEOR score introduced by (Banerjee and Lavie, 2005) is based on the harmonic mean of uni-gram precision and recall, with recall weighted higher than precision.

\subsection{Results}

Table 2 summarizes the results comparing the VOGUE framework to the previous baselines for answer verbalization. VOGUE significantly outperforms the earlier baselines for both the BLEU and METEOR scores. While for the other baselines, we perform experiments with two different inputs (Question, gold Logical Form), VOGUE is the only one that directly uses both inputs (Hybrid). As we can see, for both datasets VQuAnDa and ParaQA, all baselines perform slightly worse when receiving the question as input compared to the gold logical form. This is due to the constant input pattern templates that the logical forms have. However, this does not apply to the VANiLLa dataset since it only contains simple questions. VOGUE achieves a BLEU score of 28.76 on VQuAnDa, which is 2 points higher than the second-best BERT (LF). The same applies to the METEOR score. Regarding ParaQA, VOGUE performs slightly better than the second Convolutional (LF) on BLEU score, while on METEOR score, the margin increases to 3 points. Finally, for the VANiLLa dataset, VOGUE performs considerably better compared to other baselines.

\subsection{Ablation Study}

\section{Integration with Semantic Parsing based QA system}

The logical forms used for the results in Table 2 are the gold ones, and therefore the performance of all baselines, including our framework, is boosted. In our first ablation study, we want to perform experiments in an end-to-end manner with a semantic parsing QA system, alongside the mod- 


\begin{tabular}{l|c|c|c|c|c|c}
\hline \multirow{2}{*}{\multicolumn{1}{c}{ Models }} & \multicolumn{3}{c}{ BLEU } & \multicolumn{3}{c}{ METEOR } \\
\cline { 2 - 7 } & VQuAnDa & ParaQA & VANiLLa & VQuAnDa & ParaQA & VANiLLa \\
\hline RNN & 15.43 & 22.45 & 16.66 & 53.15 & 58.41 & 58.67 \\
Convolutional & 21.32 & 25.94 & 15.42 & 57.54 & 60.82 & 61.14 \\
Transformer & 18.37 & 23.61 & 30.80 & 56.83 & 59.63 & 62.16 \\
BERT & 22.78 & 26.12 & 31.32 & 59.28 & 62.59 & 62.96 \\
\hline VOGUE (Ours) & $\mathbf{2 5 . 7 6}$ & $\mathbf{2 8 . 4 2}$ & $\mathbf{3 3 . 1 4}$ & $\mathbf{6 4 . 6 1}$ & $\mathbf{6 7 . 5 2}$ & $\mathbf{6 3 . 6 9}$ \\
\hline
\end{tabular}

Table 3: Results of the answer verbalization with a semantic parsing QA system. VOGUE still outperforms all baselines. For the baselines we employ only the question as input, while our framework employs the similarity threshold module to determine whether a hybrid verbalization can be performed.

\begin{tabular}{l|c|c|c|c|c|c}
\hline \multirow{2}{*}{\multicolumn{1}{c}{ Ablation }} & \multicolumn{3}{c|}{ BLEU } & \multicolumn{3}{c}{ METEOR } \\
\cline { 2 - 7 } & VQuAnDa & ParaQA & VANiLLa & VQuAnDa & ParaQA & VANiLLa \\
\hline Ours & $\mathbf{2 8 . 7 6}$ & $\mathbf{3 2 . 0 5}$ & $\mathbf{3 5 . 4 6}$ & $\mathbf{6 7 . 2 1}$ & $\mathbf{6 8 . 8 5}$ & $\mathbf{6 5 . 0 4}$ \\
w/o Cross Attention & 26.24 & 30.59 & 30.94 & 64.93 & 66.16 & 62.12 \\
w/o Multi-Task Learning & 25.74 & 28.15 & 29.07 & 62.31 & 63.84 & 61.49 \\
\hline
\end{tabular}

Table 4: Ablation study results that indicate the effectiveness of cross attention and multi-task learning. The first row contains the results of the VOGUE framework when training all four modules with multi-task learning. The second and third rows selectively remove the cross attention and the multi-task learning from VOGUE. Best values in bold.

\begin{tabular}{c|ccc}
\hline \multirow{2}{*}{ Module } & \multicolumn{3}{c}{ F1-Score } \\
\cline { 2 - 4 } & VQuAnDa & ParaQA & VANiLLa \\
\hline Similarity Threshold & 64.73 & 58.55 & 98.76 \\
\hline
\end{tabular}

Table 5: Similarity threshold results for each dataset.

els, to understand our framework's superior performance. In this experiment, we train a simple, sequence-to-sequence-based semantic parser system to generate the logical forms by using the questions. As expected, the generated logical forms are not all correct, and therefore this affects the verbalization results. However, in Table 3, we can see that VOGUE still outperforms all baselines in this setting. An important role here plays the similarity threshold module, enabling a hybrid approach even in a real-world scenario. We can only use the question as input for the baselines since we do not have the gold logical forms. Here, it is also interesting that in two of the datasets, our framework outperforms the baselines with a more significant margin than before (c.f. Table 3, METEOR-VQuAnDa, METEORParaQA). Finally, Figure 3 illustrates the perplexity results, which show how well a probability distribution predicts a sample. A low perplexity indicates the probability distribution is good at predicting the sample. As we can see, our framework achieves the lowest perplexity values on all three datasets compared to other the baselines.

\section{Impact of Cross Attention and Multi-Task Learning}

Our second ablation experiment demonstrates the vitality of the cross-attention module and multitask learning strategy. We first remove the crossattention module from our framework. Instead, we only concatenate the question and logical form to generate the verbalization. As observed in Table 4, we obtain worse results compared to the original configuration of VOGUE. A simple concatenation does not interchange any information between the question and the logical form, and therefore the results are expected to be lower. The crossattention module is intentionally built to determine relevance between inputs by jointly modeling the relationship between the question words and logical form actions. Next, we train all modules independently and join them on inference to understand multi-task learning efficacy. As observed, our results have a negative impact when a multitask learning strategy is not employed.

\section{Similarity Threshold Task Analysis}

Table 5 illustrates the performance of similarity threshold module. We observe that the module performs fairly well on VQuAnDa and ParaQA 

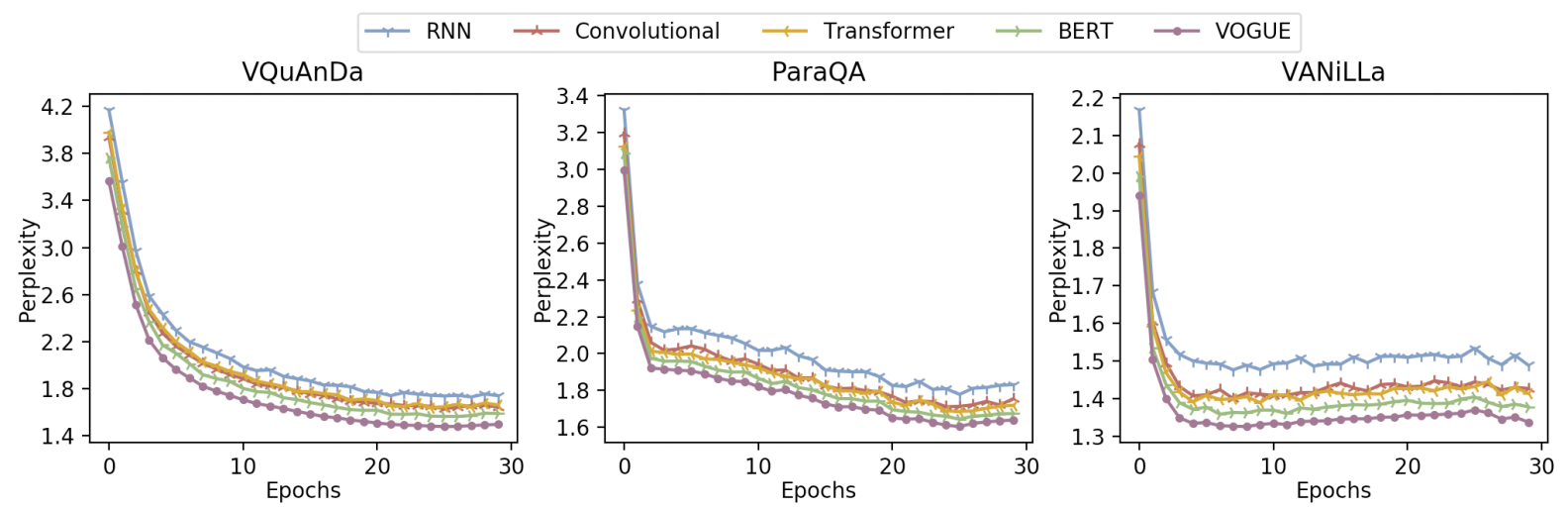

Figure 3: Perplexity curves for all three answer verbalization datasets.

with f1 scores of 64.73 and 58.55, respectively. Both datasets contain complex questions. Hence, predicting the similarity between the question and the logical form is not easy. However, as long as the module's score is beyond 50 , we are confident that using the similarity threshold module can improve our frameworks' answer verbalization results. For the VANiLLa dataset, the performance is incredibly high, with a score of 98.76. This is because the dataset contains only simple questions. Consequently, a single template pattern is employed for this dataset, and the module here has to predict if the logical form contains the correct triple relation. The task is much easier to perform compared to complex questions. Overall, the module results are pretty accurate and encourage us to apply them in our task.

\subsection{Error Analysis}

For the error analysis, we randomly sampled 100 incorrect predictions for human evaluation. We detail the reasons for two types of errors observed in the analysis:

Words Mischoose A common error of VOGUE is mischoosing a word in the answer verbalization sentence. For instance, for the question "Give me a count of everything owned by the network whose sister name is The CW?" our framework generated the answer "There are 156 television shows whose network's sister station is The CW.". However, the gold reference here is "There are 156 things whose network's sister name is The CW." As we can see, our framework misselected words in two parts of the sentence. The first one is the word "things", where it predicted "television shows". The second one is the word "name", where our model predicted "station". Both model predictions ("televi- sion shows", "station") are correlated, since they belong to the same context. Such errors do not heavily penalize the overall performance. For the example mentioned above, the BLEU and METEOR score is positive, with values 35.74 and 81.52 , respectively.

Factual Errors Another type of error of VOGUE is when it misses the semantic meaning and produces irrelevant results. It contributes to a major chunk of overall errors. There are two cases that can cause observed errors. The first one is the lack of reasoning for similar context data. When facing examples with the limited context in the dataset, the model would most definitely fail to reproduce the same context in the answer sentence. One can solve the issue by enriching the training data with other examples containing similar contexts. The second reason for having factual errors is when similarity threshold module fails to determine the inputs' relevance. As illustrated before, using the similarity threshold allows to successfully adopt a hybrid approach in a real-world scenario $(Q A+$ Answer Verbalization $)$ and exceed any previous baseline performance. Further, in the appendix, we describe the grammar used in this work, a case study, detailed experiment settings, and extended error analysis results.

\section{Conclusions}

The considered hypothesis in the paper was to study the impact of jointly utilizing the question and logical form on the answer verbalization task. We empirically observed that the proposed "hybrid" approach implemented in the VOGUE framework provides a flexibility to be deployed in a real world scenario where a QA system not always will produce the correct logical form. We 
systematically studied the impact of our choices in the proposed architecture. For instance, the ablation study demonstrates the effectiveness of multitask learning and cross-attention module. Albeit effective, VOGUE is the first step towards a more extensive research agenda. Based on our observations, we submit the following open research questions in this domain: 1) KGs have been recently used as a source of external knowledge in the tasks such as entity and relation linking, which are also prominent for question answering (Bastos et al., 2021; Mulang et al., 2020). It is yet to be studied if external knowledge from KGs or other sources may positively impact the answer verbalization. 2) There are empirical evaluations that for AI systems, the explanations regarding the retrieved answers improve trustworthiness, especially in wrong prediction (Kouki et al., 2017). Hence, how an answer verbalization can be explained remains an important open research direction. 3) In our work, we focused on English as an underlying language, and a multi-lingual approach is the next viable step.

\section{References}

Jason Armitage, Endri Kacupaj, Golsa Tahmasebzadeh, Swati, Maria Maleshkova, Ralph Ewerth, and Jens Lehmann. 2020. Mlm: A benchmark dataset for multitask learning with multiple languages and modalities. In 29th ACM CIKM. ACM.

Satanjeev Banerjee and Alon Lavie. 2005. METEOR: An automatic metric for MT evaluation with improved correlation with human judgments. In Proceedings of the ACL Workshop on Intrinsic and Extrinsic Evaluation Measures for Machine Translation and/or Summarization. ACL.

Anson Bastos, Abhishek Nadgeri, Kuldeep Singh, Isaiah Onando Mulang, Saeedeh Shekarpour, Johannes Hoffart, and Manohar Kaul. 2021. Recon: Relation extraction using knowledge graph context in a graph neural network. In Proceedings of The Web Conference $(W W W)$, page :N/A.

Debanjali Biswas, Mohnish Dubey, Md Rashad Al Hasan Rony, and Jens Lehmann. 2021. VANiLLa : Verbalized Answers in Natural Language at Large Scale. arXiv e-prints, page arXiv:2105.11407.

Antoine Bordes, Y-Lan Boureau, and Jason Weston. 2017. Learning end-to-end goal-oriented dialog. In 5th ICLR, 2017.

R. Cipolla, Y. Gal, and A. Kendall. 2018. Multi-task learning using uncertainty to weigh losses for scene geometry and semantics. In 2018 IEEE/CVF Conference on CVPR.
Jacob Devlin, Ming-Wei Chang, Kenton Lee, and Kristina Toutanova. 2019. BERT: Pre-training of deep bidirectional transformers for language understanding. In NAACL. ACL.

Basil Ell, Andreas Harth, and Elena Simperl. 2014. Sparql query verbalization for explaining semantic search engine queries. In $E S W C$.

Sébastien Ferré. 2017. Sparklis: an expressive query builder for sparql endpoints with guidance in natural language. Semantic Web.

Bin Fu, Yunqi Qiu, Chengguang Tang, Yang Li, Haiyang Yu, and Jian Sun. 2020. A survey on complex question answering over knowledge base: Recent advances and challenges. arXiv preprint arXiv:2007.13069.

Claire Gardent, Anastasia Shimorina, Shashi Narayan, and Laura Perez-Beltrachini. 2017. Creating training corpora for NLG micro-planners. In 55th ACL. ACL.

Jonas Gehring, Michael Auli, David Grangier, Denis Yarats, and Yann N. Dauphin. 2017. Convolutional sequence to sequence learning. In 34th ICML, Proceedings of Machine Learning Research. PMLR.

Endri Kacupaj, Barshana Banerjee, Kuldeep Singh, and Jens Lehmann. 2021a. Paraqa: A question answering dataset with paraphrase responses for single-turn conversation. In Eighteenth ESWC.

Endri Kacupaj, Joan Plepi, Kuldeep Singh, Harsh Thakkar, Jens Lehmann, and Maria Maleshkova. 2021b. Conversational question answering over knowledge graphs with transformer and graph attention networks. In The 16th Conference of the European Chapter of the Association for Computational Linguistics.

Endri Kacupaj, Hamid Zafar, Jens Lehmann, and Maria Maleshkova. 2020. Vquanda: Verbalization question answering dataset. In The Semantic Web. Springer International Publishing.

Firas Kassawat, Debanjan Chaudhuri, and Jens Lehmann. 2019. Incorporating joint embeddings into goal-oriented dialogues with multi-task learning. In ESWC 2019.

Diederik P Kingma and Jimmy Ba. 2015. Adam, a method for stochastic optimization. In 3rd ICLR.

Pigi Kouki, James Schaffer, Jay Pujara, John O'Donovan, and Lise Getoor. 2017. User preferences for hybrid explanations. In Proceedings of the Eleventh ACM Conference on Recommender Systems, pages 84-88.

Jens Lehmann, Robert Isele, Max Jakob, Anja Jentzsch, Dimitris Kontokostas, Pablo N. Mendes, Sebastian Hellmann, Mohamed Morsey, Patrick van Kleef, Sören Auer, and Christian Bizer. 2015. Dbpedia - a large-scale, multilingual knowledge base extracted from wikipedia. Semantic Web. 
Wenqiang Lei, Xisen Jin, Min-Yen Kan, Zhaochun Ren, Xiangnan He, and Dawei Yin. 2018. Sequicity: Simplifying task-oriented dialogue systems with single sequence-to-sequence architectures. In 56th ACL. ACL.

Jie Liu, Shaowei Chen, Bingquan Wang, Jiaxin Zhang, $\mathrm{Na} \mathrm{Li}$, and Tong $\mathrm{Xu}$. 2020. Attention as relation: Learning supervised multi-head self-attention for relation extraction. In IJCAI-20. IJCAI.

Thang Luong, Hieu Pham, and Christopher D. Manning. 2015. Effective approaches to attention-based neural machine translation. In EMNLP. ACL.

S. Mohla, S. Pande, B. Banerjee, and S. Chaudhuri. 2020. Fusatnet: Dual attention based spectrospatial multimodal fusion network for hyperspectral and lidar classification. In 2020 IEEE/CVF Conference on CVPRW.

Isaiah Onando Mulang, Kuldeep Singh, Chaitali Prabhu, Abhishek Nadgeri, Johannes Hoffart, and Jens Lehmann. 2020. Evaluating the impact of knowledge graph context on entity disambiguation models. In $C I K M$.

Kishore Papineni, Salim Roukos, Todd Ward, and WeiJing Zhu. 2002. Bleu: a method for automatic evaluation of machine translation. In 40th ACL.

Jeffrey Pennington, Richard Socher, and Christopher Manning. 2014. Glove: Global vectors for word representation. In EMNLP. ACL.

Joan Plepi, Endri Kacupaj, Kuldeep Singh, Harsh Thakkar, and Jens Lehmann. 2021. Context transformer with stacked pointer networks for conversational question answering over knowledge graphs. In Eighteenth ESWC.

Kuldeep Singh, Arun Sethupat Radhakrishna, Andreas Both, Saeedeh Shekarpour, Ioanna Lytra, Ricardo Usbeck, Akhilesh Vyas, Akmal Khikmatullaev, Dharmen Punjani, Christoph Lange, et al. 2018. Why reinvent the wheel: Let's build question answering systems together. In Proceedings of the 2018 World Wide Web Conference.

Linfeng Song, Ante Wang, Jinsong Su, Yue Zhang, Kun Xu, Yubin Ge, and Dong Yu. 2020. Structural information preserving for graph-to-text generation. In 58th ACL. ACL.

Nitish Srivastava, Geoffrey Hinton, Alex Krizhevsky, Ilya Sutskever, and Ruslan Salakhutdinov. 2014. Dropout: a simple way to prevent neural networks from overfitting. The journal of machine learning research, 15(1):1929-1958.

Christina Unger, Lorenz Bühmann, Jens Lehmann, Axel-Cyrille Ngonga Ngomo, Daniel Gerber, and Philipp Cimiano. 2012. Template-based question answering over rdf data. In Proceedings of the $21 \mathrm{st}$ international conference on World Wide Web.
Ashish Vaswani, Noam Shazeer, Niki Parmar, Jakob Uszkoreit, Llion Jones, Aidan N. Gomez, Lukasz Kaiser, and Illia Polosukhin. 2017. Attention is all you need. In NIPS.

Denny Vrandečić and Markus Krötzsch. 2014. Wikidata: A free collaborative knowledgebase. Commun. ACM.

X. Wei, T. Zhang, Y. Li, Y. Zhang, and F. Wu. 2020. Multi-modality cross attention network for image and sentence matching. In 2020 IEEE/CVF Conference on CVPR.

Ikuya Yamada, Akari Asai, Hiroyuki Shindo, Hideaki Takeda, and Yuji Matsumoto. 2020. LUKE: Deep contextualized entity representations with entityaware self-attention. In EMNLP. ACL.

Chao Zhao, Marilyn Walker, and Snigdha Chaturvedi. 2020. Bridging the structural gap between encoding and decoding for data-to-text generation. In 58th ACL. ACL.

Weiguo Zheng, Hong Cheng, Lei Zou, Jeffrey Xu Yu, and Kangfei Zhao. 2017. Natural language question/answering: Let users talk with the knowledge graph. In 2017 ACM CIKM.

\section{A Appendix}

Due to page limit, we could not put several empirical results in the main paper. This section describes the remaining empirical studies.

\section{B Grammar}

For the logical forms, we employ a grammar that can be used to capture the entire context of the question with the minimum number of actions. We prefer not to reinvent the wheel, and therefore we adopted the grammar from existing stateof-the-art question answering systems (Kacupaj et al., 2021b; Plepi et al., 2021). However, we do not employ all the actions from these works; Table 7 illustrates the complete grammar with all the defined actions that we used for all three answer verbalization datasets. As we can see, for a couple of actions, we also have their reverse occurrence (e.g. find, find_reverse). This is done to match the knowledge graph triple direction (subject-predicate-object). In some questions, we might have the subject or the object entity. Having both normal and reverse actions helps us identify the correct answer directly based on the model's predicted action. In Table 6, we illustrate how the actions can be used to annotate questions from all three datasets. For instance, for the VQuAnDa question "Which sports 


\begin{tabular}{|c|c|c|}
\hline Dataset & Question & Logical Form \\
\hline \multirow{4}{*}{ VQuAnDa } & In which team did Dave Bing and & union(find(Dave_Bing, draftteam), \\
\hline & Ron Reed started their basketball career? & find(Ron_Reed, draftteam)) \\
\hline & $\begin{array}{l}\text { Does the white river flow into } \\
\text { the connecticut river? }\end{array}$ & $\begin{array}{l}\text { is_in(find(Connecticut_River, rightTributary), } \\
\text { White_River_(Vermont)) }\end{array}$ \\
\hline & $\begin{array}{l}\text { Which sports are played in schools affiliated } \\
\text { with the Harvest Christian Center? }\end{array}$ & $\begin{array}{l}\text { find(filter_type(find_reverse(Harvest_Christian_Center, } \\
\text { religiousAffiliation), School), sport) }\end{array}$ \\
\hline \multirow{3}{*}{ ParaQA } & $\begin{array}{l}\text { How many people have been part of } \\
\text { Chicago Bulls team? }\end{array}$ & count(find_reverse(Chicago_Bulls, team)) \\
\hline & $\begin{array}{l}\text { Name the office holder whose alma mater } \\
\text { is Harvard-Westlake School and resting place } \\
\text { is Alta Mesa Memorial Park? }\end{array}$ & $\begin{array}{l}\text { intersection(filter_type(find_reverse( } \\
\text { Harvard-Westlake_School, almamater), officeholder), } \\
\text { find_reverse(Alta_Mesa_Memorial_Park, restingplace)) }\end{array}$ \\
\hline & $\begin{array}{l}\text { Name all the broadcast area of the TV stations } \\
\text { which has Rodrigues as one of the broadcast area? }\end{array}$ & $\begin{array}{l}\text { find(filter_type(find_reverse(Rodrigues, broadcastArea), } \\
\text { TelevisionStation), broadcastArea) }\end{array}$ \\
\hline \multirow{3}{*}{ VANiLLa } & $\begin{array}{l}\text { What is the nonprofit organization } \\
\text { where Bruce Bochy was educated? }\end{array}$ & filter_type(find(Q586449, P69), Q163740) \\
\hline & $\begin{array}{l}\text { Which language can Paolo Brera } \\
\text { understand? }\end{array}$ & find(Q2423068, P1412) \\
\hline & $\begin{array}{l}\text { Which administrative territory is Shaun } \\
\text { Cunnington an inhabitant of ? }\end{array}$ & filter_type(find(Q7490823, P27)) \\
\hline
\end{tabular}

Table 6: Dataset examples annotated with gold logical forms.

are played in schools affiliated with the Harvest Christian Center?" the gold logical form does include three different actions from our grammar (find, filter_type and find_reverse). Where the "find_reverse" is used to identify all the subject entities of the triple (?subject, religiousAffiliation, Harvest_Christian_Center). Another interesting example is the ParaQA question "Name the office holder whose alma mater is Harvard-Westlake School and resting place is Alta Mesa Memorial Park?" where the gold logical form contains the action "intersection" which allows us to identify the intersection between two sets of entities. Similarly, we also use the "union" action. Next, for the quantitative questions, we employ the action "count" which returns the length of the entity set. Finally, for verification questions, we have the "is_in" action, which checks whether an entity exists in a set.

\section{Case Study}

We further manually inspect our framework VOGUE outputs for conducting a case study to understand the performance better. As shown in Table 9, we find that in the first example, VOGUE can produce the exact verbalization with the reference. Here the logical form contains three actions (count and two find_reverse), and is not a simple question. Such results indicate the superb

\begin{tabular}{|c|c|}
\hline Action & Description \\
\hline set $\rightarrow$ find $(e, p)$ & $\begin{array}{l}\text { set of objects part of the triples } \\
\text { with subject } e \text { and predicate } p\end{array}$ \\
\hline set $\rightarrow$ find_reverse $(e, p)$ & $\begin{array}{l}\text { set of subjects part of the triples } \\
\text { with object } e \text { and predicate } p\end{array}$ \\
\hline set $\rightarrow$ filter_type(set, tp) & $\begin{array}{l}\text { filter the given set of entities } \\
\text { based on the given type }\end{array}$ \\
\hline $\begin{array}{l}\text { boolean } \rightarrow \text { is_in(entity, set) } \\
\text { number } \rightarrow \text { count(set) }\end{array}$ & $\begin{array}{l}\text { check if the entity is in the set } \\
\text { number of elements in the set }\end{array}$ \\
\hline set $\rightarrow$ union $\left(\right.$ set $_{1}$, set $\left._{2}\right)$ & union of set $_{1}$ and set $_{2}$ \\
\hline set $\rightarrow$ intersection $\left(\right.$ set $_{1}$, set $\left._{2}\right)$ & intersection of $s e t_{1}$ and $s e t_{2}$ \\
\hline
\end{tabular}

Table 7: Predefined grammar with respective actions to generate logical forms.

performance of our framework. Next, we can see the question "Who is the scientist whose academic advisor was Karl Ewald Hasse?" here, VOGUE manages to generate almost the exact verbalization. In particular, it only mischoses a single word, which is still relevant to the context, and the generated result could be easily considered correct. Our framework here generated the answer "The scientist whose doctoral advisor is Karl Ewald Hasse is Robert Koch." and the word it missed was "academic" where it replaced it with "doctoral". The following example illustrates the robustness of our framework. Here the question is "What are the movies with Daniel Waters as screenwriter?" and our model produces a flawless verbalization and it only complicates the words "screenwriter" and "director". It also replaces the word "films" with "movies" which can be considered synonyms 
and make no significant difference in verbalization. The generated response here is grammatically sound and adequately represent all the information in the question and logical form. Finally, in the last example, we can see that VOGUE has generalized in the answer verbalization compared to the reference. More precisely, the question here refers to cars designed by a company, and the reference also mentions it. However, our model produces a more general but at the same time fluent verbalization that refers to "things" instead of "cars", which again can be considered a valid response for the given input.

\section{Hyperparameters and Module Configurations}

Table 8 summarizes the hyperparameters used across the VOGUE framework. Starting with training parameters, we employ a batch size of 256, a learning rate of 0.001 and we train for 100 epochs. For the optimization, we use the Noam optimizer proposed by (Vaswani et al., 2017), where authors use an Adam optimizer (Kingma and $\mathrm{Ba}, 2015$ ) with several warmup steps for the learning rate. In our case, the number of warmup steps is 4000 . During optimization, we clip the gradients with a max norm of 5 . We apply a dropout with a probability 0.1 athwart our framework and use an embedding dimension of $d=$ 300. All our modules operate under the same embedding dimension. We apply the GloVe word embedding model to our input tokens with a word embedding dimension of 300 . For the transformer encoder and decoder, we use the configurations from (Vaswani et al., 2017). Our model dimension is $d=300$, with a total number of $H=6$ heads and $L=2$ layers. The inner feed-forward linear layers have dimension $d_{f f}=600,(2 *$ 300). Following the base transformer parameters, we apply residual dropout (Srivastava et al., 2014) to the summation of the embeddings and the positional encodings in both encoder and decoder stacks with a rate of 0.1 . The similarity threshold module receives an input of dimension 600 where here a linear layer is responsible for reducing it to 300 , which is the framework dimension. Next, a LeakyReLU, dropout, and a linear layer are used for the final prediction. Finally, for the cross attention module, we apply hyperparameters similar to the transformer model. Our dimension remains of size $d=300$ and again the number of heads is

\begin{tabular}{cc}
\hline Hyperparameters & Value \\
\hline epochs & 100 \\
batch size & 256 \\
learning rate & 0.001 \\
dropout ratio & 0.1 \\
optimizer & Adam \\
warmup steps & 4000 \\
clip max norm & 5 \\
$\beta_{1}$ & 0.9 \\
$\beta_{2}$ & 0.999 \\
$\varepsilon$ & $1 \mathrm{e}-09$ \\
model dim & 300 \\
word embedding model & GloVe \\
word embedding dim & 300 \\
transformer layers & 2 \\
transformer heads & 6 \\
similarity threshold & LeakyRelu \\
non-linear activation & \\
\hline
\end{tabular}

Table 8: Hyperparameters for VOGUE framework.

$H=6$. However, we do not apply multiple layers here. Likewise, dropout is applied with probability 0.1 . The number of training parameters for VQuAnDa, ParaQA, and VANiLLa datasets are $12.9 \mathrm{M}, 14.9 \mathrm{M}$, and $46.8 \mathrm{M}$ respectively.

\section{E Detailed Experiments}

We provide detailed experiment results for metrics such as perplexity, BLEU-1, BLEU-2, BLEU3, BLEU-4 and METEOR in the following Tables $10,11,12$. 


\begin{tabular}{|c|c|}
\hline Question & $\begin{array}{l}\text { How many other home stadium are there of the soccer club } \\
\text { whose home stadium is Luzhniki Stadium? }\end{array}$ \\
\hline Logical Form & $\begin{array}{l}\text { count(find_reverse(find_reverse(Luzhniki_Stadium, homeStadium), } \\
\text { homeStadium)) }\end{array}$ \\
\hline Reference & $\begin{array}{l}\text { There are } 9 \text { home stadiums of the soccer club } \\
\text { whose home stadium is Luzhniki Stadium. }\end{array}$ \\
\hline VOGUE & $\begin{array}{l}\text { There are } 9 \text { home stadiums of the soccer club } \\
\text { whose home stadium is Luzhniki Stadium. }\end{array}$ \\
\hline Question & Who is the scientist whose academic advisor was Karl Ewald Hasse? \\
\hline Logical Form & $\begin{array}{l}\text { filter_type(find_reverse(Karl_Ewald_Hasse, academicAdvisor), } \\
\text { Scientist) }\end{array}$ \\
\hline Reference & $\begin{array}{l}\text { The scientist whose academic advisor is Karl Ewald Hasse } \\
\text { is Robert Koch. }\end{array}$ \\
\hline VOGUE & $\begin{array}{l}\text { The scientist whose doctoral advisor is Karl Ewald Hasse } \\
\text { is Robert Koch. }\end{array}$ \\
\hline Question & What are the movies with Daniel Waters as screenwriter? \\
\hline Logical Form & filter_type(find_reverse(Daniel_Waters, screenplay), Film) \\
\hline Reference & $\begin{array}{l}\text { The films with the screenplay written by Daniel Waters are Batman Returns, } \\
\text { Demolition Man (film), Hudson Hawk, The Adventures of Ford Fairlane. }\end{array}$ \\
\hline VOGUE & $\begin{array}{l}\text { The movies whose director is Daniel Waters are Batman Returns, } \\
\text { Demolition Man (film), Hudson Hawk, The Adventures of Ford Fairlane. }\end{array}$ \\
\hline Question & $\begin{array}{l}\text { Which person designed the cars which has been designed by } \\
\text { ASC Creative Services? }\end{array}$ \\
\hline Logical Form & $\begin{array}{l}\text { find(filter_type(find_reverse(ASC_Creative_Services, designCompany), } \\
\text { Automobile), designer) }\end{array}$ \\
\hline Reference & $\begin{array}{l}\text { The designers of the cars whose designer company is ASC Creative } \\
\text { Services are Warren, Michigan, Michigan, ASC Creative Services. }\end{array}$ \\
\hline VOGUE & $\begin{array}{l}\text { The things which have been designed by ASC Creative Services are } \\
\text { Warren, Michigan, Michigan, ASC Creative Services. }\end{array}$ \\
\hline
\end{tabular}

Table 9: Sample output of our framework. 


\begin{tabular}{l|c|c|c|c|c|c}
\hline & \multicolumn{7}{c}{ VQuAnDa } \\
\cline { 2 - 7 } Models & Perplexity & BLEU-1 & BLEU-2 & BLEU-3 & BLEU-4 & METEOR \\
\hline RNN (Q) & 1.8132 & 50.71 & 35.11 & 21.65 & 15.43 & 53.15 \\
RNN (LF) & 1.7297 & 54.38 & 40.17 & 23.12 & 20.19 & 57.06 \\
Convolutional (Q) & 1.6696 & 58.49 & 42.86 & 27.87 & 21.32 & 57.54 \\
Convolutional (LF) & 1.6170 & 62.58 & 44.79 & 30.43 & 26.02 & 64.30 \\
Transformer (Q) & 1.6953 & 57.27 & 40.22 & 26.92 & 18.37 & 56.83 \\
Transformer (LF) & 1.6434 & 60.34 & 44.38 & 31.45 & 23.18 & 60.17 \\
BERT (Q) & 1.6474 & 59.78 & 43.64 & 28.25 & 20.78 & 59.28 \\
BERT (LF) & 1.5575 & 62.20 & 45.27 & 32.83 & 24.96 & 65.92 \\
\hline VOGUE (ours) $(\mathbf{H})$ & $\mathbf{1 . 4 7 9 1}$ & $\mathbf{6 5 . 9 7}$ & $\mathbf{4 9 . 2 0}$ & $\mathbf{3 6 . 7 1}$ & $\mathbf{2 8 . 7 6}$ & $\mathbf{6 7 . 2 1}$ \\
\hline
\end{tabular}

Table 10: Detailed results on VQuAnDa dataset.

\begin{tabular}{l|c|c|ccc|c}
\hline & \multicolumn{6}{c}{ ParaQA } \\
\cline { 2 - 7 } Models & Perplexity & BLEU-1 & BLEU-2 & BLEU-3 & BLEU-4 & METEOR \\
\hline RNN (Q) & 1.8816 & 58.56 & 40.64 & 28.74 & 22.45 & 58.41 \\
RNN (LF) & 1.7768 & 61.87 & 44.48 & 31.86 & 26.36 & 61.87 \\
Convolutional (Q) & 1.7879 & 60.15 & 43.29 & 30.15 & 25.94 & 60.82 \\
Convolutional (LF) & 1.7056 & 65.93 & 49.95 & 37.98 & 31.89 & 65.85 \\
Transformer (Q) & 1.7701 & 59.68 & 42.22 & 30.33 & 23.61 & 59.63 \\
Transformer (LF) & 1.6731 & 64.04 & 47.73 & 36.62 & 28.01 & 63.75 \\
BERT (Q) & 1.7217 & 61.21 & 45.69 & 32.29 & 24.12 & 62.59 \\
BERT (LF) & 1.6459 & $\mathbf{6 6 . 4 7}$ & 50.91 & 37.61 & 29.31 & 65.92 \\
\hline VOGUE (ours) (H) & $\mathbf{1 . 6 0 4 3}$ & $\mathbf{6 7 . 6 0}$ & $\mathbf{5 1 . 9 4}$ & $\mathbf{3 9 . 9 4}$ & $\mathbf{3 2 . 0 5}$ & $\mathbf{6 8 . 8 5}$ \\
\hline
\end{tabular}

Table 11: Detailed results on ParaQA dataset.

\begin{tabular}{|c|c|c|c|c|c|c|}
\hline \multirow[b]{2}{*}{ Models } & \multicolumn{6}{|c|}{ VANiLLa } \\
\hline & Perplexity & BLEU-1 & BLEU-2 & BLEU-3 & BLEU-4 & METEOR \\
\hline RNN (Q) & 1.5629 & 41.29 & 30.85 & 22.76 & 16.66 & 58.67 \\
\hline RNN (LF) & 1.4708 & 42.33 & 29.71 & 23.16 & 16.45 & 55.34 \\
\hline Convolutional (Q) & 1.4715 & 40.45 & 28.39 & 23.38 & 15.42 & 61.14 \\
\hline Convolutional (LF) & 1.4043 & 42.10 & 29.53 & 22.34 & 16.89 & 58.72 \\
\hline Transformer (Q) & 1.4683 & 55.09 & 40.77 & 35.62 & 30.80 & 62.16 \\
\hline Transformer (LF) & 1.3817 & 53.37 & 39.28 & 34.98 & 28.12 & 59.01 \\
\hline $\operatorname{BERT}(\mathrm{Q})$ & 1.4193 & 57.23 & 43.83 & 37.47 & 31.32 & 62.96 \\
\hline BERT (LF) & 1.3557 & 56.03 & 42.59 & 36.22 & 30.11 & 59.27 \\
\hline VOGUE (ours) (H) & 1.3261 & 62.72 & 48.77 & 40.80 & 35.14 & 65.04 \\
\hline
\end{tabular}

Table 12: Detailed results on VANiLLa dataset. 